\title{
A Multilayered Box Model for Calculating Preliminary Remediation Goals in Soil Screening
}

\author{
Chao Shan, Iraj Javandel \\ Earth Sciences Division \\ Lawrence Berkeley National Laboratory \\ Berkeley, CA 94720, USA
}

\begin{abstract}
In the process of screening a soil against a certain contaminant, we define the health-risk based preliminary remediation goal (PRG) as the contaminant concentration above which some remedial action may be required. PRG is thus the first standard (or guidance) for judging a site. An over-estimated PRG (a too-large value) may cause us to miss some contaminated sites that can threaten human health and the environment. An under-estimated PRG (a too-small value), on the other hand, may lead to unnecessary cleanup and waste tremendous resources. The PRGs for soils are often calculated on the assumption that the contaminant concentration in soil does not change with time. However, that concentration usually decreases with time as a result of different chemical and transport mechanisms. The static assumption thus exaggerates the long-term exposure dose and results in a too-small PRG. We present a box model that considers all important transport processes and obeys the law of mass conservation. We can use the model as a tool to estimate the transient contaminant concentrations in air, soil and groundwater. Using these concentrations in conjunction with appropriate health risk
\end{abstract}


parameters, we may estimate the PRGs for different contaminants. As an example, we calculated the tritium PRG for residential soils. The result is quite different from, but within the range of, the two versions of the corresponding PRG previously recommended by the U.S. EPA.

Key Words: box model, soil screening, preliminary remediation goal (PRG), tritium.

\section{Introduction}

Most subsurface contaminations originate from chemical releases at the ground surface or in the vadose zone. Depending on their chemical properties, the transport and fate for different contaminants vary greatly. Volatile organic compounds (VOCs) can descend with infiltration water and ascend through gas-phase advection and diffusion. Most heavy metals, on the other hand, can only adsorb to soil particles, dissolve into soil water, and be transported into the groundwater via percolation. In addition, radionuclides and some other chemicals can decay with time, changing into different chemicals. The kind, magnitude, extent, and distribution of contamination at a given site usually determine the degree of potential threat to human health and the environment.

To aid in the nationwide efforts to identify problem sites, the United States Environmental Protection Agency (U.S. EPA) has spent tremendous time and efforts ${ }^{1-2}$ in providing the Soil Screening Guidance (SSG) for both qualitatively analyzing contaminant conditions at different sites and quantitatively calculating PRGs for different contaminants under different scenarios. As a risk-based tool, SSG plays an important role in expediting site investigation and cleanup. Today, the SSG and other relevant 
information are available on the EPA website where additional tools, such as the PRG calculator, are also provided.

There is no doubt that EPA's efforts have made the SSG more accessible and convenient to a wide-range of people, from consulting firms to regulatory agencies. There are some developments in the SSG, however, that are sources of concern to both site-owners and the surrounding community. For example, the PRG of tritium for residential soil was in the range of 10,200 to $14,000 \mathrm{pCi} / \mathrm{g}$ in the EPA 1991 document $^{3}$, but was listed as $2.3 \mathrm{pCi} / \mathrm{g}$ on the EPA website in February 2003: an astounding four orders of magnitude lower. EPA later removed the new PRG for tritium (vapor) and left it open on its website.

The old (1991) EPA document ${ }^{3}$ calculated the radionuclide (including tritium) PRGs for residential soil by considering only two pathways (i.e., ingestion of soil and external exposure). The new equation on the EPA website considers two additional pathways: inhalation of dust and volatiles, and ingestion of garden produce. The new equation also considers radioactive decay. With these changes, the new PRG equation seems to make more sense. However, the new equation has not resolved the two old problems: (a) the calculated PRG is still unrelated to the size and extent of the contamination, and (b) the new equation still does not consider the reduction of contaminant mass in the soil caused by transport processes.

The first problem is rather explicit: size matters. At the same level of contaminant concentration, a football-sized volume of contaminated soil will surely not pose a threat as big as that posed by the extent of soil contamination covering a football field. When we use the EPA equation to calculate the PRGs, we assume that sufficient 
amount of contaminant exists in the soil for the period of exposure (30 years for residents). The validity of this assumption depends on the size and extent of the soil contamination.

The second problem is less obvious. However, if we consider a conservative (non-decaying, non-reactive) contaminant, the problem becomes apparent. Here the EPA equation actually assumes that the contaminant concentration in soil will stay constant, which is not the case for most sites where the contamination source has been removed. The transport process on these sites is characterized as the one of "redistribution". As a simple example of redistribution, suppose a site originally has $4 \mathrm{~kg}$ of certain conservative volatile contaminant in soil, and if later $1 \mathrm{~kg}$ of the contaminant vaporizes into the atmosphere, other $1 \mathrm{~kg}$ is carried by percolation water down to the aquifer, and another $1 \mathrm{~kg}$ is taken up by the plants, there should be only $1 \mathrm{~kg}$ of contaminant left in the vadose zone soil.

Assumptions and approximations are usually essential in the soil screening process. For example, the contaminant transport is usually a transient, three-dimensional process, but is assumed to be one-dimensional in the SSG. Also, the contaminant concentration can hardly be uniform in nature, but we use a mean value to represent the spatially varying concentration in an exposure medium. These assumptions, in general, lead to a smaller PRG that is more health-protective. We accept these assumptions for problem simplification. We, however, should not accept any assumptions or treatments that violate physical laws. The new PRG equations still violate the law of mass conservation in that they never take account the reduction of contaminant concentration in soil due to dissolution, volatilization, percolation, diffusion, and plant-root extraction. 
As a result, the new PRG equations end with much smaller PRGs. Therefore, there is a need to find a more realistic mathematical model to calculate the transient contaminant concentrations in different media (air, soil, groundwater, and vegetation).

Mathematical models of different degrees of complexity are available for predicting the fate and transport of chemicals in the subsurface (e.g., Jury et al. ${ }^{4}, 1990$; Shan and Stephens $\left.{ }^{5}, 1995\right)$. Shan and Stephens ${ }^{5}$ (1995) presented the analytical solutions for vertical transport of VOC in a homogeneous vaodse zone of finite thickness. The transport is originated from an initially uniform VOC contamination within a certain depth interval in the vadose zone. The analytical solutions calculate the transient VOC concentration profiles, as well as the mass flux rates to the aquifer and the atmosphere. By setting the Henry's constant to zero, the solutions should also be applicable to nonvolatile chemicals. These analytical solutions are certainly useful in estimating the contaminant concentrations in the exposure media, which provide the critical input information for health-risk assessment. Simple models, however, are still needed in soil screening for two main reasons: (1) soil screening is based on risk assessment that does not require a detailed transport process but a rough picture of the contamination levels at different exposure media; and (2) simple models can provide such a rough picture much more conveniently. This paper presents a simple model for soil screening and demonstrates its application in calculating the tritium PRG for residential soil. Although the current PRG formulae recommended by the EPA do not consider the exposure pathway of groundwater ingestion, here we also derive the solution for contaminant concentration in groundwater for potential future applications. 


\section{Theory}

Box models, the simplest finite-difference models, have been used in health risk assessment (e.g., McKone et al. $\left.{ }^{6}, 1997\right)$, and in the prediction of chemical concentration in layered soils (e.g., McKone and Bennett ${ }^{7}, 2003$ ). Let us consider a box model composed of an air box on the top, a groundwater box at the bottom, and a soil box in the middle. To better simulate a non-uniform soil contamination, we further divide the soil box (see Figure 1) into two zones: an initially contaminated zone (the $\mathrm{C}$ zone) above, and an initially clean buffer zone (the B zone) below. Our purpose is to use the model to calculate the approximate distribution of the contaminant in soil, air, and groundwater with time.

For any given contaminated site, we first approximate the areal extent of contamination by a rectangle with one side along the groundwater flow direction. We then take a unit-thick slice along that direction (Figure 1) for the study. We use lower case letters to represent the box dimensions: $l[\mathrm{~L}]$ the box-length, and $h[\mathrm{~L}]$ the boxheights. We use upper case letters to represent other characteristics of the system: $B$ $[\mathrm{L} / \mathrm{T}]$ the average soil-breathing rate, $\mathrm{C}\left[\mathrm{M} / \mathrm{L}^{3}\right]$ the concentration at time $t[\mathrm{~T}\}, F$ $\left[\mathrm{M} / \mathrm{L}^{2} / \mathrm{T}\right]$ (diffusive contaminant mass flux rate), $G[\mathrm{~L} / \mathrm{T}]$ the average groundwater flow velocity, $P[\mathrm{~L} / \mathrm{T}]$ the average percolation rate, $V[\mathrm{~L} / \mathrm{T}]$ the average vegetation waterconsumption rate, and $W[\mathrm{~L} / \mathrm{T}]$ the average wind speed. Additionally, we use subscripts $a, c, b$, and $g$ to designate the parameters for the air, contaminated, buffer, and groundwater zones, respectively. We simply call this model the ACBG box model. The above symbols are shown in Figure 1.

We make the following assumptions for the model development. 
- The vegetation can be treated as a sink term in the contaminated zone.

- The concentration of a chemical in indoor air is the same as that in outdoor air.

- Although wind frequently changes its direction, the mixing effects are the same for wind in different directions, i.e., it always carries clean air into the air box, and contaminated air out of it. We thus use an average wind speed to represent the wind effect.

- An additional flux-term, the evapotranspiration, $E_{T}[\mathrm{~L} / \mathrm{T}]$, is also needed only for tritium in the form of tritiated water (HTO).

- All contaminant releases have been stopped at the site.

We will also assume that the following transport modes are negligible: (a) the contaminant mass transported by surface runoff, (b) the diffusive mass flux from the air box to the surrounding atmosphere, and (c) the human intake of contaminant mass. Neglecting these processes usually results in an overestimate of the contaminant concentrations inside the four boxes.

Although the soil box is divided into two zones, both zones are assumed to have the same percolation rate $(P)$, as well as the same soil properties. As indicated above, we use $C_{c}$ and $C_{b}$ to represent the contaminant concentrations in the contaminated and buffer zones, respectively. These concentrations are total concentrations that represent the amount of contaminant per unit volume of soil, including soil particles, soil water, and soil gas. Correspondingly, the contaminant concentration in soil water (SW) and soil gas (SG) were calculated by (Shan and Stephens $\left.{ }^{5}, 1995\right)$ :

$$
\begin{array}{ll}
C_{c}^{S W}=C_{c} / R_{W} ; & C_{b}^{S W}=C_{b} / R_{W} \\
C_{c}^{S G}=C_{c} / R_{G} ; & C_{b}^{S G}=C_{b} / R_{G}
\end{array}
$$


The dimensionless coefficients, $R_{W}$ and $R_{G}$, in the above equations are:

$$
\begin{gathered}
R_{W}=\rho_{b} K_{S W}+\theta+(\phi-\theta) K_{H}+\rho_{b} K_{H} K_{S G} \\
R_{G}=R_{W} / K_{H}
\end{gathered}
$$

In (2a), $\rho_{b}\left[\mathrm{M} / \mathrm{L}^{3}\right]$ is the dry soil bulk density, $\phi$ the porosity, $\theta$ the volumetric water content, $K_{H}$ the dimensionless Henry's constant. $K_{S W}\left[\mathrm{~L}^{3} / \mathrm{M}\right]$ and $K_{S G}\left[\mathrm{~L}^{3} / \mathrm{M}\right]$ are the solid-water and solid-gas distribution coefficients, respectively.

\section{Differential Equations}

Using the above assumptions and parameters, we now develop the differential equations for estimating the contaminant concentrations in different boxes at any given time $t[\mathrm{~T}]$.

\section{A. The Air box}

In Figure 1, if we apply the law of mass conservation to the contaminant in the air box, we obtain:

$$
-\left[W h_{a} C_{a}+l h_{a} \lambda C_{a}-l E_{T} \frac{C_{c}}{R_{W}}+l\left(B+\frac{2 D}{h_{c}}\right)\left(C_{a}-\frac{C_{c}}{R_{G}}\right)\right] \Delta t=\left(C_{a, \Delta t}-C_{a}\right) l h_{a}
$$

The right-hand side of (3) represents the net mass change in the air box over a small time period, $\Delta t[\mathrm{~T}]$. The change is due to the different processes given on the lefthand side of (3). Among them, the first term $-W h_{a} C_{a} \Delta t$ represents the contaminant mass taken out of the air box by wind in $\Delta t$; the second term $-l h_{a} \lambda C_{a} \Delta t$ represents the reduction of contaminant mass in the air box due to decay in $\Delta t$; the third term $l E_{T}\left(C_{d} / R_{W}\right) \Delta t$ is for tritiated water only, it represents the tritium mass added to the air box via evapotranspiration in $\Delta t$; and the last term represents the contaminant mass change in 
the air box in $\Delta t$ due to gas advection and diffusion between the air box and the contaminated zone. In addition to the parameters defined above, the effective diffusion coefficient in soil, $D\left[\mathrm{~L}^{2} / \mathrm{T}\right]$, can be estimated using the Millington ${ }^{8}(1959)$ model. Note that we have used $h_{c} / 2$ as the length of diffusive path between the contaminated zone and air box. Since the diffusion coefficient in air is orders of magnitude larger than that in soil gas, contaminant transport through gas diffusion from soil to the air box is essentially controlled by diffusion in soil. We thus arrange (3) to reflect this phenomenon. However, the diffusive fluxes in a box model are always underestimated by using an averaged (smaller) concentration gradient. As a result, the concentration in the $\mathrm{C}$ zone will always be overestimated, which is more conservative because this zone is the major contributor to health risk through dermal contact and ingestion of soil and garden produce.

In (3), $C_{a, \Delta t}\left[\mathrm{M} / \mathrm{L}^{3}\right]$ is the contaminant concentration in the air box at time $t+\Delta t$. Rearranging (3) and taking the limit for $\Delta t \rightarrow 0$ we obtain the differential equation for the air box:

$$
\frac{d C_{a}}{d t}=-\lambda_{a} C_{a}+\lambda_{1} C_{c}
$$

where $\lambda_{a}\left[\mathrm{~T}^{-1}\right]$ and $\lambda_{1}\left[\mathrm{~T}^{-1}\right]$ are two constants defined by

$$
\begin{aligned}
& \lambda_{a}=\lambda+\frac{W}{l}+\frac{B h_{c}+2 D}{h_{a} h_{c}} \\
& \lambda_{1}=\frac{B h_{c}+2 D}{R_{G} h_{a} h_{c}}+\frac{E_{T}}{R_{W} h_{a}}
\end{aligned}
$$

The first term in (5a), $\lambda\left[\mathrm{T}^{-1}\right]$, is the decay constant. The second term in (5b) represents evapotranspiration and vanishes for all chemicals but not for tritiated water vapor. 


\section{B. The Contaminated Zone}

If the contaminant concentration in this zone at time $t+\Delta t$ is represented by $C_{c, \Delta t}$ $\left[\mathrm{M} / \mathrm{L}^{3}\right]$, by applying the law of mass conservation, we obtain the following equation:

$$
-\left[\left(P+V+E_{T}\right) l \frac{C_{c}}{R_{w}}+l h_{c} C_{c} \lambda+l\left(B+\frac{2 D}{h_{c}}\right)\left(\frac{C_{c}}{R_{G}}-C_{a}\right)+\frac{2 D l\left(C_{c}-C_{b}\right)}{R_{G}\left(h_{b}+h_{c}\right)}\right] \Delta t=\left(C_{c, \Delta t}-C_{c}\right) l h_{c}
$$

Similar to (3), the right-hand side of (6) represents the net mass change in the contaminated zone over a small time period, $\Delta t$. The change is due to the different processes given on the left-hand side of (6). Among them, the first term $-\left(P+V+E_{T}\right) l\left(C_{d} / R_{W}\right) \Delta t$ represents the contaminant mass taken out of contaminated zone by percolation, vegetation up-take, and evapotranspiration (for HTO only); the second term $-l h_{c} \lambda C_{c} \Delta t$ represents the reduction of contaminant mass in the contaminated zone due to decay; the third term represents the contaminant mass change in the contaminated zone due to gas advection and diffusion between the air box and the contaminated zone; the last term represents the contaminant mass change in the contaminated zone due to gas diffusion between the buffer zone and the contaminated zone.

Rearranging (6) and taking the limit for $\Delta t \rightarrow 0$, we obtain the following differential equation for the contaminated zone:

$$
\frac{d C_{c}}{d t}=-\lambda_{c} C_{c}+\lambda_{2} C_{a}+\lambda_{3} C_{b}
$$

where $\lambda_{c}\left[\mathrm{~T}^{-1}\right], \lambda_{2}\left[\mathrm{~T}^{-1}\right]$, and $\lambda_{3}\left[\mathrm{~T}^{-1}\right]$ are three constants defined by

$$
\lambda_{c}=\lambda+\frac{P+V+E_{T}}{h_{c} R_{W}}+\frac{B h_{c}+2 D}{h_{c}^{2} R_{G}}+\frac{2 D}{h_{c}\left(h_{b}+h_{c}\right) R_{G}}
$$




$$
\begin{aligned}
& \lambda_{2}=\frac{B h_{c}+2 D}{h_{c}^{2}} \\
& \lambda_{3}=\frac{2 D}{h_{c}\left(h_{b}+h_{c}\right) R_{G}}
\end{aligned}
$$

\section{The Buffer Zone}

If the contaminant concentration in the buffer zone at time $t+\Delta t[\mathrm{~T}]$ is represented by $C_{b, \Delta t}\left[\mathrm{M} / \mathrm{L}^{3}\right]$, by applying the law of mass conservation to the contaminant in the buffer zone, we obtain the following equation:

$$
-\left[P l \frac{C_{b}-C_{c}}{R_{w}}+l h_{b} C_{b} \lambda+\frac{2 D l\left(C_{b}-C_{c}\right)}{\left(h_{b}+h_{c}\right) R_{G}}\right] \Delta t=\left(C_{b, \Delta t}-C_{b}\right) l h_{b}
$$

The three terms on the left-hand side in (9) represent the mass changes in the buffer zone due to percolation, decay, and gas diffusion, respectively. Rearranging (9) and taking the limit for $\Delta t \rightarrow 0$, we obtain the following differential equation for the buffer zone:

$$
\frac{d C_{b}}{d t}=-\lambda_{b} C_{b}+\lambda_{4} C_{c}
$$

where $\lambda_{b}\left[\mathrm{~T}^{-1}\right]$, and $\lambda_{4}\left[\mathrm{~T}^{-1}\right]$ are two constants defined by

$$
\begin{aligned}
& \lambda_{b}=\lambda+\frac{P}{h_{b} R_{W}}+\frac{2 D}{h_{b}\left(h_{b}+h_{c}\right) R_{G}} \\
& \lambda_{4}=\frac{P}{h_{b} R_{w}}+\frac{2 D}{h_{b}\left(h_{b}+h_{c}\right) R_{G}}
\end{aligned}
$$




\section{The Groundwater box}

The solution for the groundwater box is developed here for potential future applications. Since we do not consider any contaminant transport from the groundwater back to the vadose zone, the equation for this box does not affect the equations for the air and soil boxes, and thus can be solved independently. The equation based on mass conservation is:

$$
\left(P l \frac{C_{b}}{R_{w}}-C_{g} R n \lambda h_{g} l-C_{g} h_{g} G\right) \Delta t=\left(C_{g, \Delta t}-C_{g}\right) R n h_{g} l
$$

where $C_{g, \Delta t}\left[\mathrm{M} / \mathrm{L}^{3}\right]$ is the contaminant concentration in groundwater at time $t+\Delta t[\mathrm{~T}] ; n$ the porosity of the aquifer; and $R$ the retardation factor that is calculated by:

$$
R=1+\rho_{g} K_{d} / n
$$

where $\rho_{g}\left[\mathrm{M} / \mathrm{L}^{3}\right]$ is the dry soil bulk density of the aquifer material; and $K_{d}\left[\mathrm{~L}^{3} / \mathrm{M}\right]$ the distribution coefficient. The three terms on the left-hand side in (12) represent the mass changes in the groundwater box due to percolation, decay, and groundwater advection, respectively. Rearranging (12) and taking the limit for $\Delta t \rightarrow 0$ we obtain the differential equation for the groundwater box:

$$
\frac{d C_{g}}{d t}=-\lambda_{g} C_{g}+\lambda_{5} C_{b}
$$

where $\lambda_{g}\left[\mathrm{~T}^{-1}\right]$ and $\lambda_{5}\left[\mathrm{~T}^{-1}\right]$ are two constants defined by

$$
\begin{aligned}
& \lambda_{g}=\lambda+\frac{G}{R n l} \\
& \lambda_{5}=\frac{P}{R R_{W} n h_{g}}
\end{aligned}
$$




\section{$\underline{\text { Initial Conditions }}$}

We use an initial condition at the time right after the release of contaminants into the vadose zone has stopped and assume a uniform contaminant distribution in the contaminated zone, i.e.

$$
\begin{aligned}
& C_{c}(0)=C_{0} \\
& C_{a}(0)=C_{b}(0)=C_{g}(0)=0
\end{aligned}
$$

where $C_{0}\left[\mathrm{M} / \mathrm{L}^{3}\right]$ is a constant representing the average initial contaminant concentration in the contaminated zone.

\section{$\underline{\text { Solutions }}$}

Solving (4), (7), (10), and (14) simultaneously against the initial conditions (16a) and (16b), we obtain the final solutions for transient contaminant concentrations in the four boxes (see Appendix A for detailed derivations) as follows:

$$
\begin{gathered}
C_{a}=C_{0}\left(a_{1} e^{-k_{1} t}+a_{2} e^{-k_{2} t}+a_{3} e^{-k_{3} t}\right) \\
C_{c}=C_{0}\left(c_{1} e^{-k_{1} t}+c_{2} e^{-k_{2} t}+c_{3} e^{-k_{3} t}\right) \\
C_{b}=C_{0}\left(b_{1} e^{-k_{1} t}+b_{2} e^{-k_{2} t}+b_{3} e^{-k_{3} t}\right) \\
C_{g}=C_{0}\left(g_{1} e^{-k_{1} t}+g_{2} e^{-k_{2} t}+g_{3} e^{-k_{3} t}+g_{4} e^{-\lambda_{g} t}\right)
\end{gathered}
$$

The thirteen dimensionless coefficients are defined by:

$$
\begin{aligned}
& a_{1}=\frac{\lambda_{1}\left(\lambda_{b}-k_{1}\right)}{\left(k_{1}-k_{2}\right)\left(k_{1}-k_{3}\right)} \\
& a_{2}=\frac{\lambda_{1}\left(\lambda_{b}-k_{2}\right)}{\left(k_{2}-k_{1}\right)\left(k_{2}-k_{3}\right)} \\
& a_{3}=\frac{\lambda_{1}\left(\lambda_{b}-k_{3}\right)}{\left(k_{3}-k_{1}\right)\left(k_{3}-k_{2}\right)}
\end{aligned}
$$




$$
\begin{aligned}
& c_{1}=\frac{k_{1}^{2}-\left(\lambda_{a}+\lambda_{b}\right) k_{1}+\lambda_{a} \lambda_{b}}{\left(k_{1}-k_{2}\right)\left(k_{1}-k_{3}\right)} \\
& c_{2}=\frac{k_{2}^{2}-\left(\lambda_{a}+\lambda_{b}\right) k_{2}+\lambda_{a} \lambda_{b}}{\left(k_{2}-k_{1}\right)\left(k_{2}-k_{3}\right)} \\
& c_{3}=\frac{k_{3}^{2}-\left(\lambda_{a}+\lambda_{b}\right) k_{3}+\lambda_{a} \lambda_{b}}{\left(k_{3}-k_{1}\right)\left(k_{3}-k_{2}\right)} \\
& b_{1}=\frac{\lambda_{4}\left(\lambda_{a}-k_{1}\right)}{\left(k_{1}-k_{2}\right)\left(k_{1}-k_{3}\right)} \\
& b_{2}=\frac{\lambda_{4}\left(\lambda_{a}-k_{2}\right)}{\left(k_{2}-k_{1}\right)\left(k_{2}-k_{3}\right)} \\
& b_{3}=\frac{\lambda_{4}\left(\lambda_{a}-k_{3}\right)}{\left(k_{3}-k_{1}\right)\left(k_{3}-k_{2}\right)} \\
& g_{1}=\frac{\lambda_{4} \lambda_{5}\left(k_{1}-\lambda_{a}\right)}{\left(k_{1}-k_{2}\right)\left(k_{1}-k_{3}\right)\left(k_{1}-\lambda_{g}\right)} \\
& g_{2}=\frac{\lambda_{4} \lambda_{5}\left(k_{2}-\lambda_{a}\right)}{\left(k_{2}-k_{1}\right)\left(k_{2}-k_{3}\right)\left(k_{2}-\lambda_{g}\right)} \\
& g_{3}=\frac{\lambda_{4} \lambda_{5}\left(k_{3}-\lambda_{a}\right)}{\left(k_{3}-k_{1}\right)\left(k_{3}-k_{2}\right)\left(k_{3}-\lambda_{g}\right)} \\
& g_{4}=\frac{\lambda_{4} \lambda_{5}\left(\lambda_{g}-\lambda_{a}\right)}{\left(\lambda_{g}-k_{1}\right)\left(\lambda_{g}-k_{2}\right)\left(\lambda_{g}-k_{3}\right)}
\end{aligned}
$$

In the Appendix we give the formulae for calculating the three constants $k_{1}, k_{2}$, and $k_{3}$. The above solutions can easily be converted to the corresponding ones for relative concentration $\left(C_{r}\right)$ in a box (i.e., $C_{r a}=C_{a} / C_{0} ; C_{r c}=C_{c} / C_{0} ; C_{r b}=C_{b} / C_{0}$; and $C_{r g}=$ $C_{g} / C_{0}$ ), which are functions of time $t$ only. Additionally, the solutions are also implicitly dependent on nine constants $\left(\lambda_{a}, \lambda_{b}, \lambda_{c}, \lambda_{g}, \lambda_{1}, \lambda_{2}, \lambda_{3}, \lambda_{4}\right.$, and $\left.\lambda_{5}\right)$ that will be called 
attenuation constants and have the dimensions of reciprocal time $\left[\mathrm{T}^{-1}\right]$. In fact, these constants reflect relevant system characteristics and the site geometry.

Note that the above formulae are invalid if their denominators become zero. However, in most cases, the denominators of the above formulae are non-zero. Also note that the solutions given in (17a) through (17d) must be non-negative. In other words, solutions are only valid before contaminants are completely depleted. We call the time period for the concentration in the contaminant zone to reach zero the depletion-time.

\section{Results and Discussions}

One advantage of the solutions presented here is their simplicity. The only function in all solutions is the exponential type. As a result, one can easily evaluate the solutions on a spreadsheet. Let us first apply the solutions for a general case where the effect of evapotranspiration is negligible. A set of parameters used for this case is given in Table 1 to represent a "base" case.

The calculated relative concentrations in the air box, the contaminated zone, the buffer zone, and the groundwater box during a 30-year period are shown in Figure 2a, where we used a normal coordinate for time and a logarithmic coordinate for the relative concentrations. The contaminant concentrations in the air box, the buffer zone, and the groundwater box increase from zero to a maximum value and then start to decrease. The concentration in the buffer zone remains the highest at times later than three years.

To study the effect of decay, we kept all input parameters in Table 1 unchanged but varied the decay constant, $\lambda$, from zero to 1 year $^{-1}$. Results are shown in Figure $2 b$. It is clearly evident that, by increasing the decay constant, contaminant concentrations in all 
boxes decrease more rapidly. We also see the same high rate of contaminant decline due to an increase of the diffusion coefficient by 10 times over the base case (Figure 2c). The only difference is that the concentration in the air box increases to a higher maximum value at early time due to the increase of diffusion. There is a common character for all curves in Figures $2 \mathrm{a}$ through $2 \mathrm{c}$, i.e., the four curves become parallel straight lines at large times. The reason is that the first term in all solutions dominates as time becomes large.

We conducted sensitivity analyses against all parameters and summarized the results in Table 2, where the top row lists the four relative concentrations, and the first column lists the 17 input parameters. We use IS to represents inversely sensitive (the relative concentration decreases with the increase of the parameter); PS for proportionally sensitive (the relative concentration increases with the increase of the parameter); $\mathrm{S}$ for sensitive (the relative concentration varies with the variation of the parameter); and $\mathrm{x}$ for non-sensitive (the change of the relative concentration is very insignificant). For example, an increase of wind speed $W$ causes the contaminant concentration in the air box to decrease (see the IS in Table 2) but does not have any noticeable impact on the concentrations in other boxes (see the $\mathrm{x}$ in Table 2). For a larger value of $R_{w}$, there is less contamination in soil water [see (1a)]; and as a result the contaminant concentration is higher in the contaminated zone as well as in the air box (see the PS in Table 2). The concentration in the buffer zone is sensitive to $R_{w}$ (see the $\mathrm{S}$ in Table 2): for a larger $R_{w}$, it is lower at early times (because less contaminant mass is carried into the buffer zone through percolation from the contaminated zone) but higher at later times (because higher concentration maintained in the contaminated zone and less 
contaminant mass is carried out through percolation to the groundwater). However, a larger $R_{w}$ always causes a smaller concentration in groundwater (see the IS in Table 2).

\section{Applications for Tritium}

The capability of the ACBG model in estimating the transient average contaminant concentrations in air, soil, and groundwater is useful in soil screening. As an application example, we now use the above solutions to estimate the tritium PRG in residential soils based on an excess cancer risk of one out of a million. The applications to other chemicals and other receptors are similar.

Since the average concentrations in different exposure media are transient, the total dose will be calculated by integration over the exposure period, $t_{e}$ (i.e., the smaller value of the depletion-time and 30 years for residents). Theoretically, the ingestion of groundwater should be an exposure pathway in the PRG calculation. However, the current EPA formula only considers the other four exposure pathways: inhalation, ingestion of soil, ingestion of vegetation produce, and external exposure. For the PRG calculation, we introduce four intake rates: $I R_{s}$ (rate of soil ingestion, in g/year), $I R_{v}$ (rate of vegetation produce ingestion, in g/year), $I R_{e}$ (rate of soil external exposure, in g/year), and $I R_{i}$ (rate of inhalation, in $\mathrm{m}^{3} / \mathrm{year}$ ). Here we also simplify the calculation by neglecting the reduction factors used in the inhalation and external exposure pathways (U.S. EPA ${ }^{2}$, 2002), which will result in a smaller calculated PRG (more healthprotective). The equation for the target risk (TR) becomes

$$
T R=\int_{0}^{t_{e}}\left(k_{c} C_{c}+k_{a} C_{a}\right) d t
$$

where 


$$
\begin{gathered}
k_{c}=\left(S F_{s} \cdot I R_{s}+S F_{e} \cdot I R_{e}+S F_{v} \cdot I R_{v} \cdot T F_{p}\right) / \rho_{b} \\
k_{a}=S F_{i} \cdot I R_{i}
\end{gathered}
$$

In (23a) and (23b) the $S F_{j} \mathrm{~s}(j=s, e, v$, and $i$, in unit of $1 / \mathrm{pCi})$ are the slope factors for different exposure pathways; and $T F_{p}$ is the soil-to-plant transfer factor that is chemical-specific. We want to note two issues here. First, in (17a) through (17c) the concentrations are in units of mass per unit volume, while in the applications to radionuclides all concentrations need to be changed to radioactivity (e.g., pCi) per unit volume. Second, since all ingestion rates in (23a) are in the unit of g/year we have used the soil bulk density, $\rho_{b}$ as a conversion factor. Substituting (17a) and (17b) into (22), integrating the right-hand side of (22) and rearranging the resulting equation, we obtain:

$$
\begin{gathered}
C_{0}=\frac{T R}{d_{1}\left(1-e^{-k_{1} t_{e}}\right)+d_{2}\left(1-e^{-k_{2} t_{e}}\right)+d_{3}\left(1-e^{-k_{3} t_{e}}\right)} \\
d_{1}=\frac{k_{a} a_{1}+k_{c} c_{1}}{k_{1}} \\
d_{2}=\frac{k_{a} a_{2}+k_{c} c_{2}}{k_{2}} \\
d_{3}=\frac{k_{a} a_{3}+k_{c} c_{3}}{k_{3}}
\end{gathered}
$$

Equation (24a) gives $C_{0}$, the volume-based contaminant concentration in soil. To convert it to the PRG as a weight-based concentration, we once again need to use the soil bulk density $\rho_{b}$ as the conversion factor, i.e.

$$
P R G=C_{0} / \rho_{b}
$$

We now use the formulae to calculate the tritium PRG for residential soil. From the EPA database, $S F_{e}=0$ for tritium, thus eliminating the second term in (23a). All 
other parameters are listed in Table 3. For a target (cancer) risk of $T R=10^{-6}$, the calculated tritium PRG in residential soils is 289 pCi/g.

Among the 22 parameters listed in Table 3 the top five are tritium-specific, where the slope factors $\left(S F_{s}, S F_{v}\right.$, and $\left.S F_{i}\right)$ for cancer-risk and the soil-to-plant transfer factor $\left(T F_{p}\right)$ for tritium are recommended by EPA. Since tritium has a half-life of 12.3 years, the decay constant $\lambda=\ln 2 / 12.3=0.056$ year $^{-1}$. The values for three intake rates $\left(I R_{s}, I R_{v}\right.$, and $\left.I R_{i}\right)$ in Table 3 are default numbers recommended by EPA.

In Table 3 we use $h_{a}=2 \mathrm{~m}, h_{c}=1 \mathrm{~m}, h_{b}=4 \mathrm{~m}$, and $l=30 \mathrm{~m}$. We assume an average wind speed of $10^{8} \mathrm{~m} /$ year (about $11 \mathrm{~km} /$ hour or $3 \mathrm{~m} / \mathrm{s}$ ) and an annual percolation rate of $P=0.1 \mathrm{~m} /$ year. The vegetation water consumption rate $V$ should be both site- and vegetation-specific. We believe that $V$ should also affect the soil-to-plant transfer factor $\left(T F_{p}\right)$, which is a subject for future research. We assume $V=0.3 \mathrm{~m} / \mathrm{year}$ and $E_{T}=1.0$ $\mathrm{m} /$ year based on the fact that the irrigation rate of a typical garden is about 1 inch (2.54 $\mathrm{cm}$ ) per week (or $1.3 \mathrm{~m} / \mathrm{year}$ ). We also assume a soil-breathing rate $(B)$ equal to 10 $\mathrm{m} /$ year, which roughly corresponds to the case of a $10^{-8} \mathrm{~m} / \mathrm{s}$ of pneumatic conductivity for soil and a one percent of change for the atmospheric pressure. We estimated the rest of the parameters in Table $3\left(D, R_{W}\right.$, and $\left.R_{G}\right)$ as follows.

We first assume that the average soil temperature is $10{ }^{\circ} \mathrm{C}$ and that the humidity of soil gas keeps at its maximum capacity $\left(9 \mathrm{~g} / \mathrm{m}^{3}\right.$ at $\left.10{ }^{\circ} \mathrm{C}\right)$. Since tritium exists mainly in the form of tritiated water (HTO), it is reasonable to assume that the ratio of $\mathrm{HTO} / \mathrm{H}_{2} \mathrm{O}$ is the same for water vapor in soil gas as that for soil water. Since HTO is a kind of water, it is a convention to assume a zero adsorption for HTO, i.e.,

$$
K_{S W}=K_{S G}=0
$$


If we take the density of water roughly as $10^{6} \mathrm{~g} / \mathrm{m}^{3}$, we may derive the dimensionless Henry's constant for HTO as:

$$
K_{H}=9 / 10^{6}=9 \times 10^{-6}
$$

Using $\phi=0.38, \theta=0.26$, and the parameters given in (26) and (27) we obtain the numbers $R_{W}=0.26$ and $R_{G}=28,890$ shown in Table 3 . The estimation of the effective diffusion coefficient in soil is a bit tedious and we only present the main steps here. We first used the formula given in Reid et al. ${ }^{9}$ (1987) and calculated the molecular diffusion coefficient for water vapor in air, which is about $2.8 \times 10^{-5} \mathrm{~m}^{2} / \mathrm{s}$. We then applied the Millington $^{8}$ (1959) model and calculated the effective diffusion coefficient in soil, $D=$ $1.65 \times 10^{-7} \mathrm{~m}^{2} / \mathrm{s}=5.2 \mathrm{~m}^{2} /$ year.

In Table 3 the dry soil bulk density $\left(\rho_{b}\right)$ is a default value used in most health risk calculations. The integration interval, $t_{e}=2.15$ year (for this case), is the calculated time when the relative tritium concentration in the $\mathrm{C}$ zone is in the order of $10^{-8}$. Figure 3 shows the variations of the relative concentrations in air and the surface soil. The solutions in (17a) and (17b) at $t>2.15$ years in this case give negative values in small magnitude, and the integration over a period of 30 years will result in a slightly larger PRG. The difference, however, is insignificant in this case.

Further studies reveal that the contribution to the risk is mainly from the ingestion of garden produce. In Table 4 we show the PRG of tritium corresponding to different ingestion rates of garden produce $I R_{v}$. When we cut that rate in Table 3 by half $(12,740$ g/year), the calculated PRG increases to $542 \mathrm{pCi} / \mathrm{g}$; i.e., it almost doubles $(542 / 289=$ 1.88). The PRG for the same situation but without the consumption of garden produce is 4390 pCi/g (Table 4). 


\section{Conclusions}

The currently used preliminary remediation goals (PRGs) for soil screening were calculated based on the assumption of a constant contaminant concentration in soil, which is only valid for very limited cases. The applications of the PRGs to most sites where contaminant release has ended will result in a violation of the mass conservation law. A box model, ACBG, based on the law of mass conservation considers all major transport processes and thus provides more reliable results. The ACBG solutions predict the transient contaminant concentrations in air, soil and groundwater, and thus are useful tools for calculating the PRGs used in soil screening.

The ACBG -based tritium PRG for residential soils is more than a hundred times larger than that given on the EPA website previously (a new PRG for tritium as vapor is still unavailable on the EPA website), which will have a significant impact on nationwide site investigation and cleanup efforts, and save tremendous amount of taxpayers' money. The ACBG solutions also provide the convenience for potential future addition of the ingestion of groundwater as an exposure pathway.

\section{Acknowledgment}

This paper was developed at the Lawrence Berkeley National Laboratory, which is operated by the University of California for the U.S. Department of Energy under contract DE-AC03-76SF00098. The authors would like to thank Drs. M. Lippmann, T.E. McKone, and N. Goldstein for reviewing the manuscript and making constructive suggestions. 


\section{References}

1. U.S. EPA (1996). Soil Screening Guidance: Technical Background Document, $2^{\text {nd }}$ ed., EPA/540/R95/128, Office of Solid Waste and Emergency Response, Washington, DC.

2. U.S. EPA (2002). Supplemental Guidance for Developing Soil Screening Levels for Superfund Sites, OSWER 9355.4-24, Office of Solid Waste and Emergency Response, Washington, DC.

3. U.S. EPA (1991). Risk Assessment Guidance for Superfund: Volume I - Human Health Evaluation Manual (Part B, Development of Risk-based Preliminary Remediation Goals), Interim, Publication 9285.7-01B, Office of Emergency and Remedial Response, Washington, DC.

4. Jury, W. A., D. Russo, G. Streile, and H. E. Abd (1990). Evaluation of volatilization by organic chemicals residing below the soil surface, Water Resour. Res., 26(1): $13-20$.

5. Shan, C. and D. B. Stephens (1995). An analytical solution for vertical transport of volatile chemicals in the vadose zone, J. Cont. Hydrol., 18: 259-277.

6. McKone, T. E., K. P. Brand, and C. Shan (1997). Environmental Health-Risk Assessment for Tritium Releases at the National Tritium Labeling Facility at Lawrence Berkeley National Laboratory, LBL-37760.

7. McKone, T. E., and D. H. Bennett (2003). Chemical-specific representation of air-soil exchange and soil penetration in regional multimedia models, Environ. Sci. Technol. 37: 3123-3132. 
8. Millington, R. J. (1959). Gas diffusion in porous media, Science, 130: 100-102.

9. Reid, R. C., J. M. Prausnitz, and B. E. Poling (1987) The Properties of Gases and Liquids, McGraw-Hill, New York.

10. Erdelyi, A. (1954). Tables of Integral Transforms, McGraw-Hill, New York. 
Table 1. Input data used for the base case

\begin{tabular}{|c|c|}
\hline Soil-breathing rate $(B)$ & $0 \mathrm{~m} / \mathrm{year}$ \\
\hline Groundwater velocity $(G)$ & $100 \mathrm{~m} /$ year \\
\hline Percolation rate $(P)$ & $0.1 \mathrm{~m} /$ year \\
\hline Vegetation water-consumption rate $(V)$ & $0.1 \mathrm{~m} /$ year \\
\hline Wind speed (W) & $10^{8} \mathrm{~m} /$ year \\
\hline Evapotranspiration rate $\left(E_{T}\right)$ & $0 . \mathrm{m} /$ year \\
\hline Effective diffusion coefficient in soil $(D)$ & $1 \mathrm{~m}^{2} /$ year \\
\hline Decay constant $(\lambda)$ & 0 year $^{-1}$ \\
\hline Coefficient for concentration in soil water $\left(R_{W}\right)$ & 1.0 \\
\hline Coefficient for concentration in soil gas $\left(R_{G}\right)$ & 2.0 \\
\hline Retardation factor in aquifer $(R)$ & 1.0 \\
\hline Aquifer porosity $(n)$ & 0.25 \\
\hline Height of air box $\left(h_{a}\right)$ & $5 \mathrm{~m}$ \\
\hline Height of buffer zone $\left(h_{b}\right)$ & $4 \mathrm{~m}$ \\
\hline Height of contamination zone $\left(h_{c}\right)$ & $1 \mathrm{~m}$ \\
\hline Height of groundwater box $\left(h_{g}\right)$ & $5 \mathrm{~m}$ \\
\hline Box length $(l)$ & $30 \mathrm{~m}$ \\
\hline
\end{tabular}


Table 2. Sensitivity of relative concentrations to input parameters

\begin{tabular}{|c|c|c|c|c|}
\hline \multirow[b]{2}{*}{ Parameter } & \multicolumn{4}{|c|}{ Relative Concentration } \\
\hline & $C_{r a}$ & $C_{r c}$ & $C_{r b}$ & $C_{r g}$ \\
\hline$h_{a}$ & IS & $\mathrm{x}$ & $\mathrm{x}$ & $\mathrm{x}$ \\
\hline$h_{b}$ & IS & IS & IS & IS \\
\hline$h_{c}$ & PS & PS & PS & PS \\
\hline$h_{g}$ & $\mathrm{x}$ & $\mathrm{x}$ & $\mathrm{x}$ & IS \\
\hline$l$ & PS & $\mathrm{x}$ & $\mathrm{x}$ & PS \\
\hline$B$ & $\mathrm{~S}$ & IS & IS & IS \\
\hline$G$ & $\mathrm{x}$ & $\mathrm{x}$ & $\mathrm{x}$ & IS \\
\hline$P$ & IS & IS & $S$ & $S$ \\
\hline$V$ & IS & IS & IS & IS \\
\hline$W$ & IS & $\mathrm{x}$ & $\mathrm{x}$ & $\mathrm{x}$ \\
\hline$E_{T}$ & $\mathrm{~S}$ & IS & IS & IS \\
\hline$D$ & $\mathrm{~S}$ & IS & IS & IS \\
\hline$\lambda$ & IS & IS & IS & IS \\
\hline$R_{W}$ & PS & PS & $\mathrm{S}$ & IS \\
\hline$R_{G}$ & IS & PS & $S$ & PS \\
\hline$R$ & $\mathrm{x}$ & $\mathrm{x}$ & $\mathrm{x}$ & PS \\
\hline$n$ & $\mathrm{x}$ & $\mathrm{x}$ & $\mathrm{x}$ & $\mathrm{x}$ \\
\hline
\end{tabular}

Note: IS = inversely sensitive; PS = proportionally sensitive; $\mathrm{S}=$ sensitive; $\mathrm{x}=$ non-sensitive. 
Table 3. Parameters for calculating tritium PRG in residential soils

\begin{tabular}{|c|c|c|}
\hline$S F_{s}=2.2 \times 10^{-13}(1 / \mathrm{pCi})$ & $S F_{v}=1.44 \times 10^{-13}(1 / \mathrm{pCi})$ & $S F_{i}=1.99 \times 10^{-13}(1 / \mathrm{pCi})$ \\
\hline$T F_{p}=4.8$ & $\lambda=0.056\left(\mathrm{year}^{-1}\right)$ & $\rho_{b}=1.7 \times 10^{6}\left(\mathrm{~g} / \mathrm{m}^{3}\right)$ \\
\hline$I R_{s}=42(\mathrm{~g} /$ year$)$ & $I R_{v}=25,480(\mathrm{~g} / \mathrm{year})$ & $I R_{i}=6,300\left(\mathrm{~m}^{3} /\right.$ year $)$ \\
\hline$h_{a}=2(\mathrm{~m})$ & $h_{c}=1(\mathrm{~m})$ & $h_{b}=4(\mathrm{~m})$ \\
\hline$l=30(\mathrm{~m})$ & $W=10^{8}(\mathrm{~m} /$ year $)$ & $B=10(\mathrm{~m} /$ year $)$ \\
\hline$P=0.1(\mathrm{~m} /$ year $)$ & $E_{T}=1.0(\mathrm{~m} /$ year $)$ & $V=0.3(\mathrm{~m} /$ year $)$ \\
\hline$R_{W}=0.26$ & $R_{G}=28,890$ & $D=5.2\left(\mathrm{~m}^{2} /\right.$ year $)$ \\
\hline$t_{e}=2.15($ year $)$ & & \\
\hline
\end{tabular}


Table 4. Comparison of calculated PRGs for four different garden produce ingestion rates

\begin{tabular}{|c|c|c|c|c|}
\hline Ingestion rate, $I R_{v}$ (g/year) & 25,480 & 12,740 & 6370 & 0 \\
\hline PRG $(\mathrm{pCi} / \mathrm{g})$ & 289 & 542 & 964 & 4390 \\
\hline
\end{tabular}




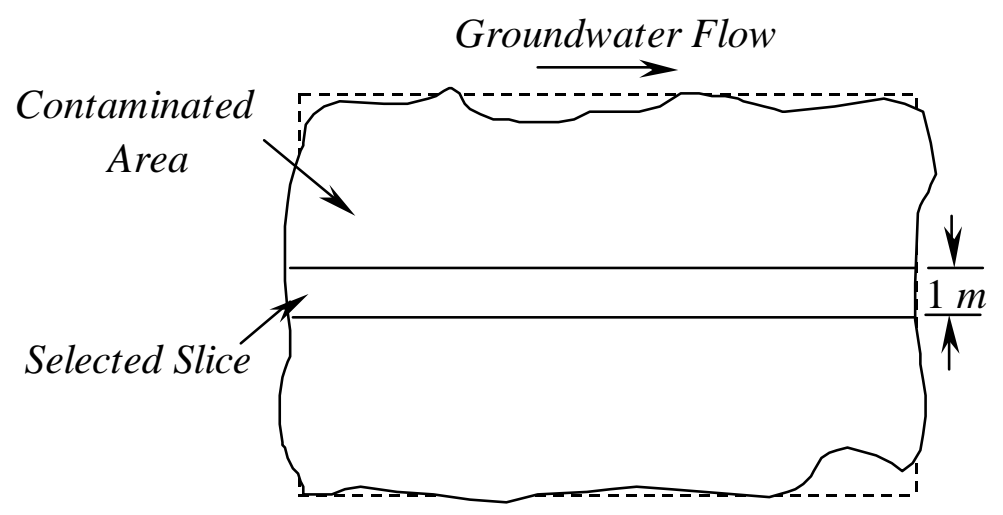

\section{Plan View}

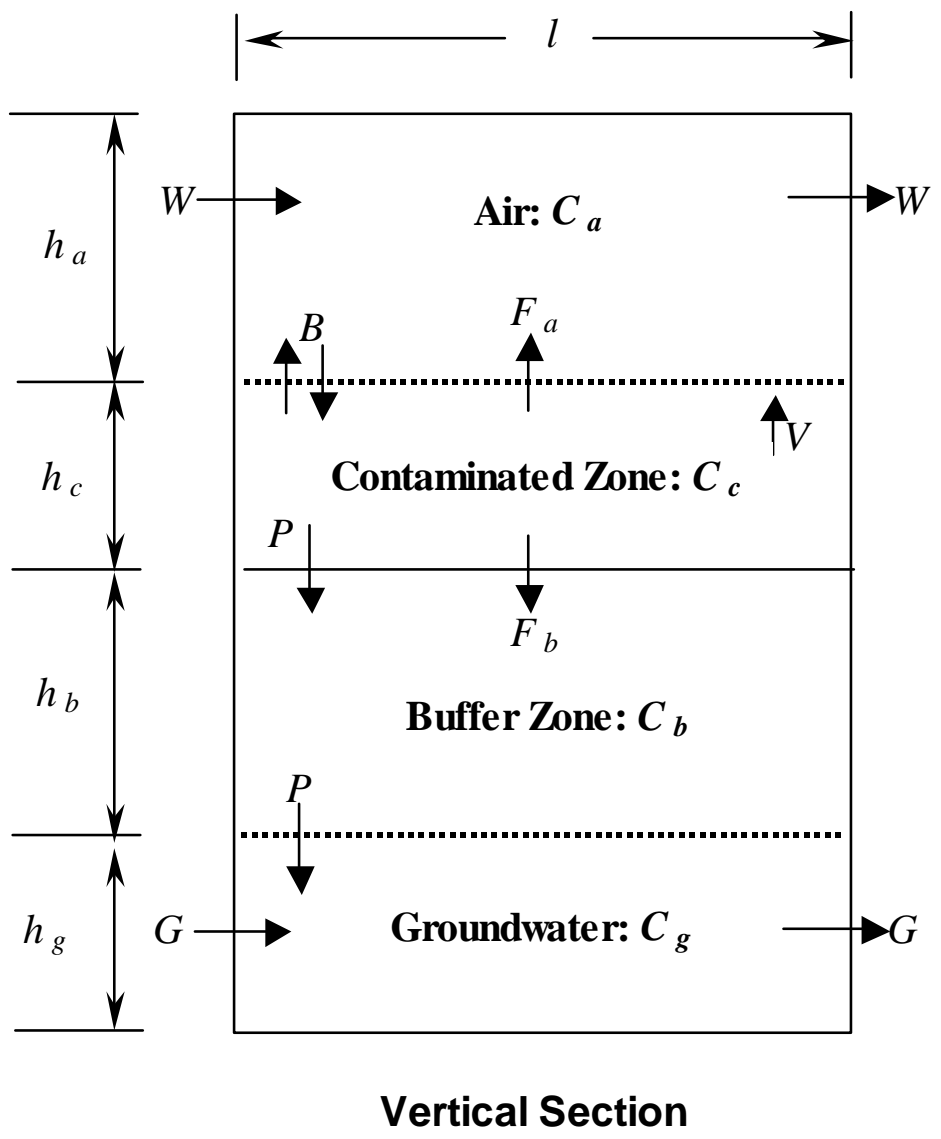

Figure 1. Schematic diagram of the ACBG box-model (see text for symbols) 


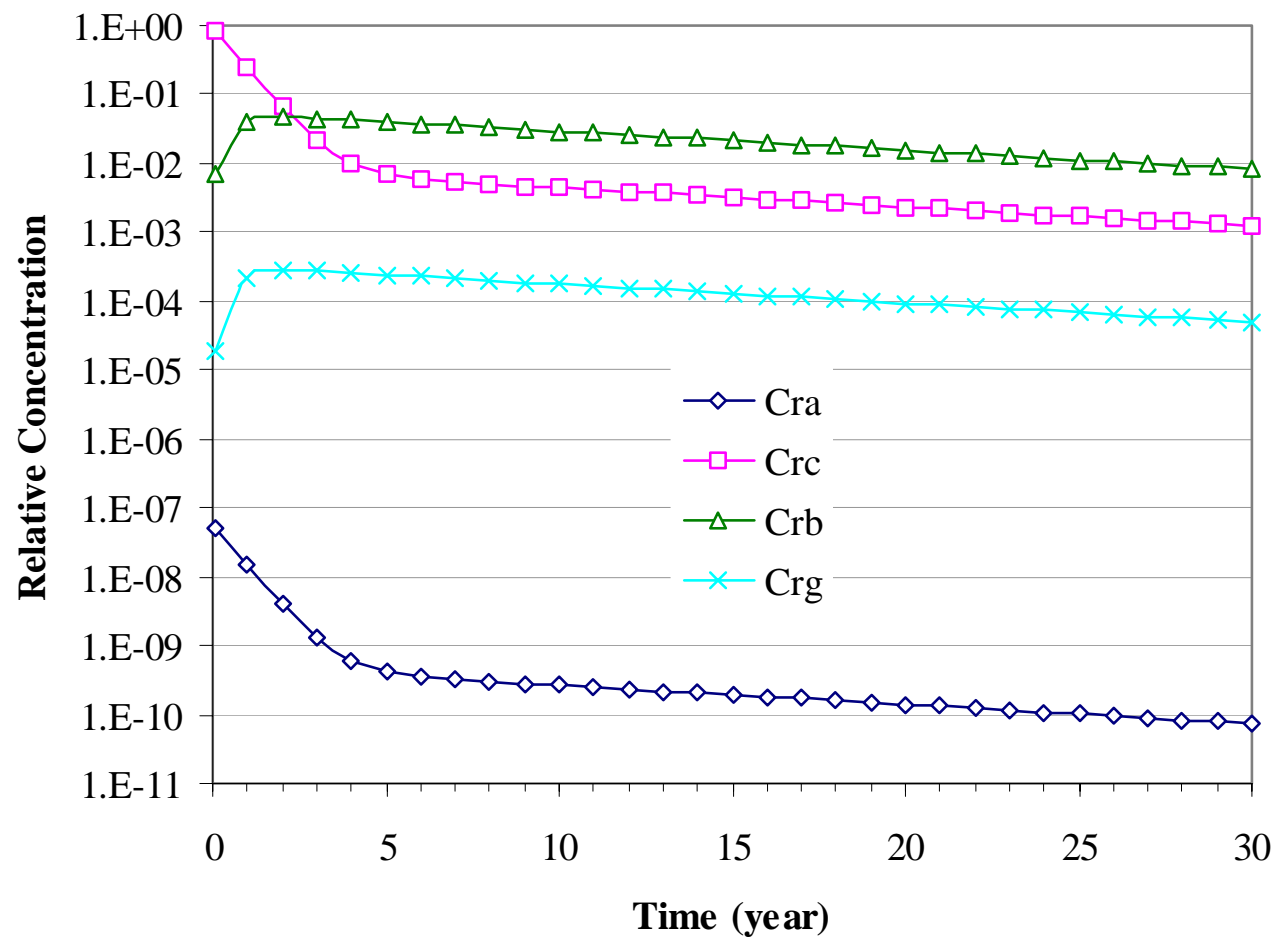

Figure 2a. Variations of relative concentrations in four boxes for the base case 


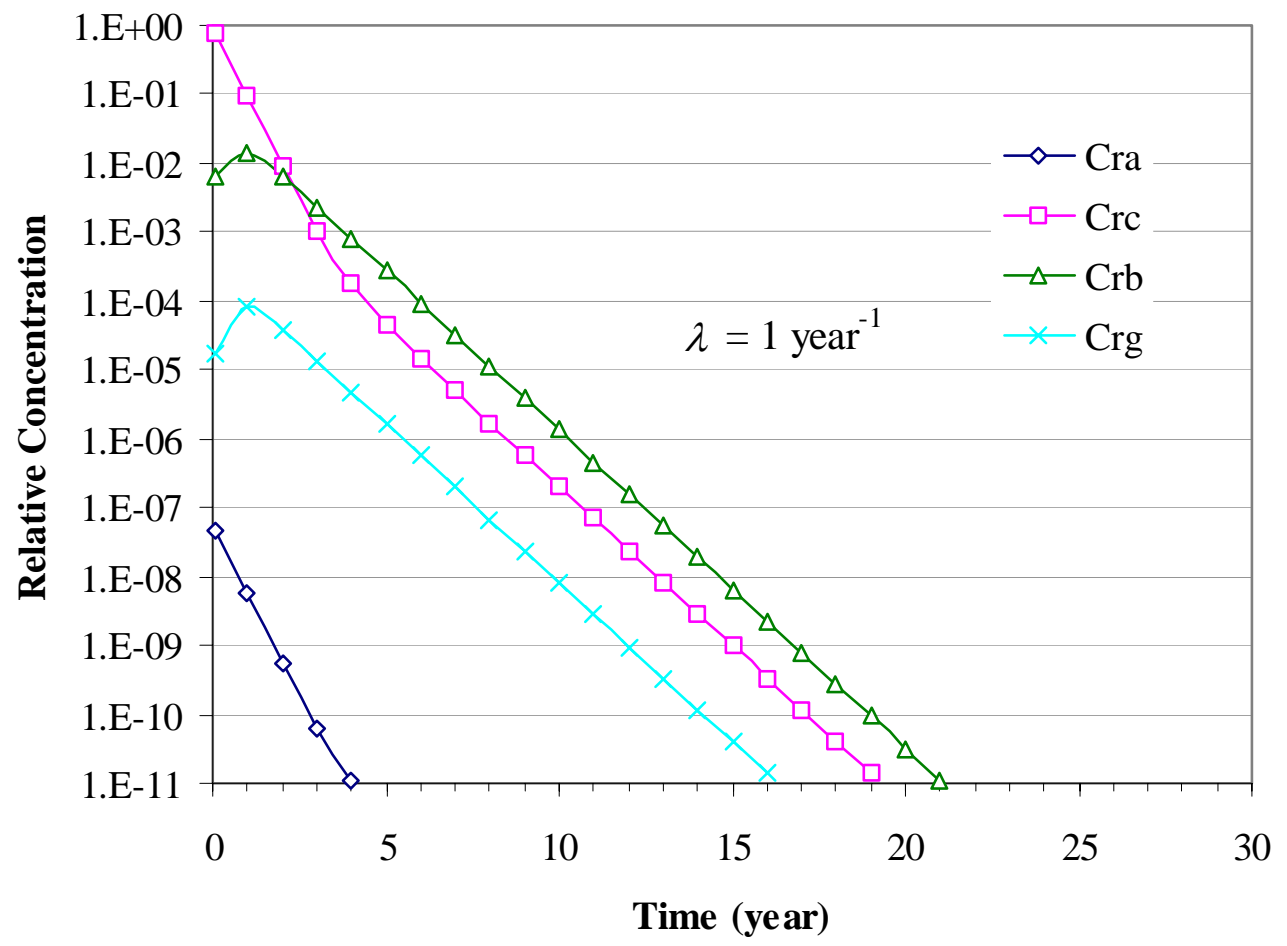

Figure $2 \mathrm{~b}$. Variations of relative concentrations for revised base case: decay effect 


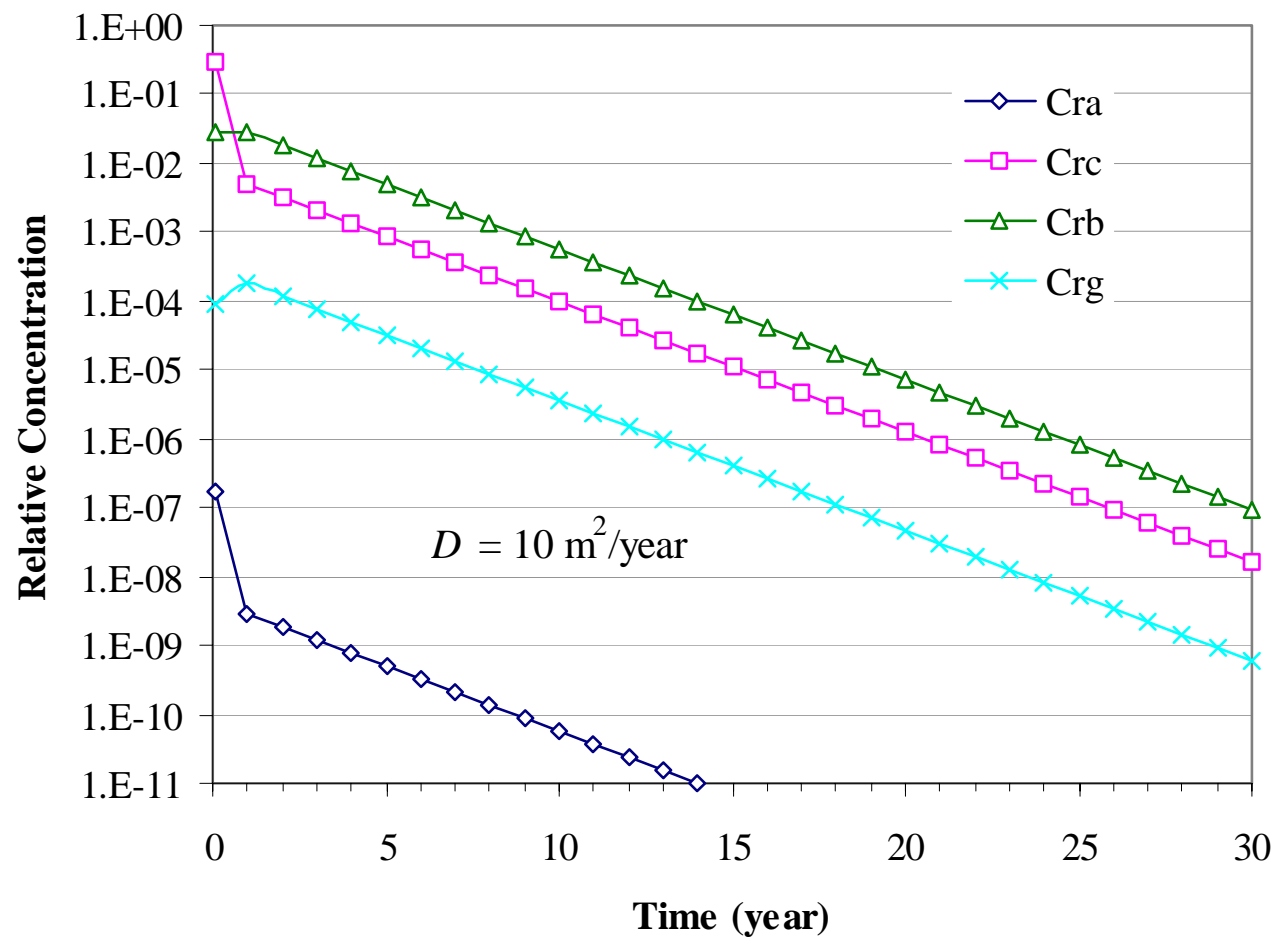

Figure 2c. Variations of relative concentrations for revised base case: diffusion effect 


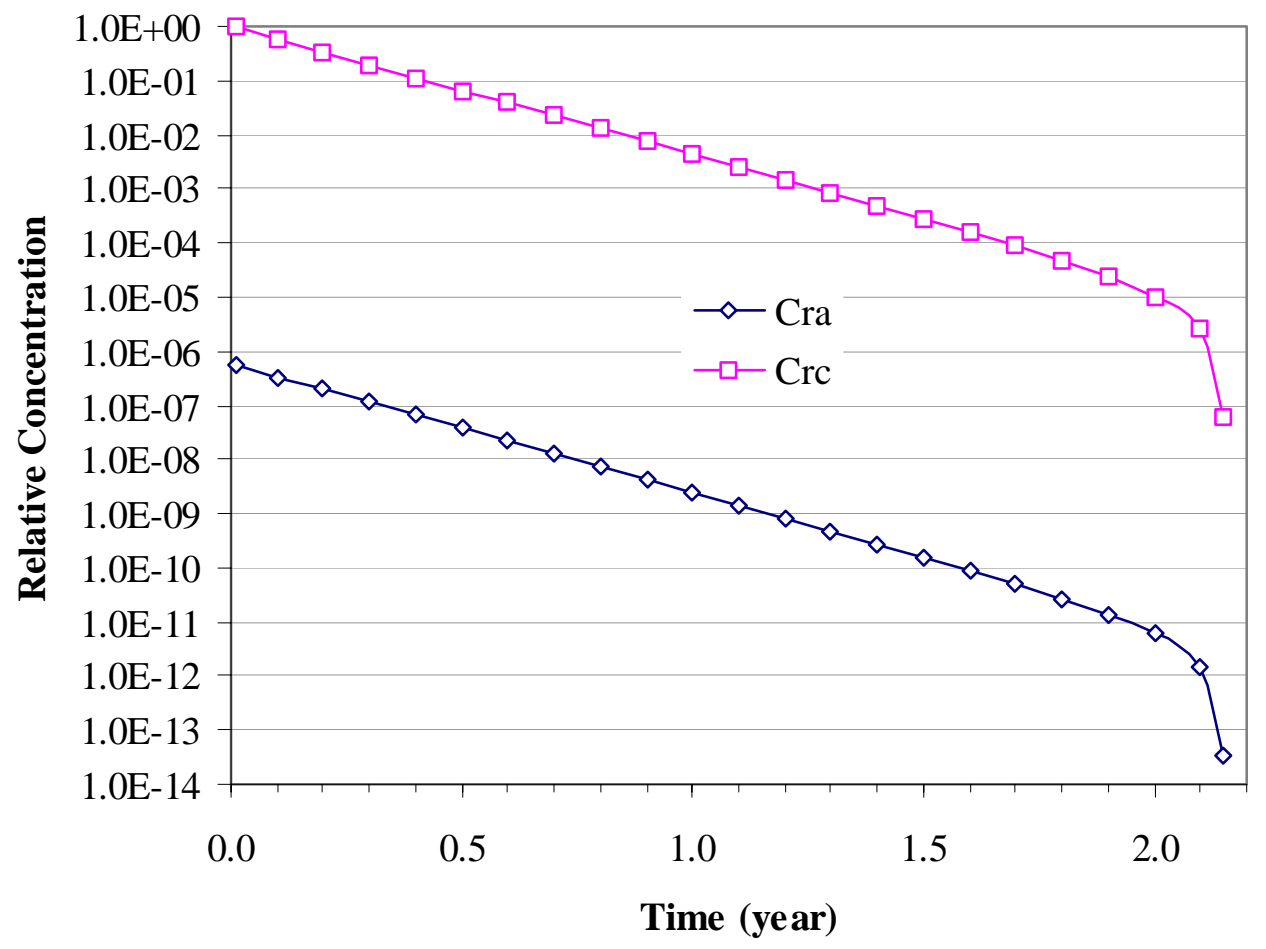

Figure 3. Relative HTO concentrations in air and surface soil 


\section{Appendix A. Solution Derivation}

For the differential equations (4), (7), (10), and (14), if we take the Laplace transform with respect to $t$ and use the initial conditions in (16a) and (16b) we obtain:

$$
\begin{aligned}
& p w_{a}=-\lambda_{a} w_{a}+\lambda_{1} w_{c} \\
& p w_{c}-C_{0}=-\lambda_{c} w_{c}+\lambda_{2} w_{a}+\lambda_{3} w_{b} \\
& p w_{b}=-\lambda_{b} w_{b}+\lambda_{4} w_{c} \\
& p w_{g}=-\lambda_{g} w_{g}+\lambda_{5} w_{b}
\end{aligned}
$$

In the above equations, $p\left[\mathrm{~T}^{-1}\right]$ is the variable for the Laplace transform; $w_{a}, w_{c}$, $w_{b}$, and $w_{g}$ are the solutions in the Laplace domain for the air box, the contaminated zone, the buffer zone, and the groundwater box, respectively. Solving (A1a) through (A1d) simultaneously, we obtain:

$$
\begin{array}{r}
w_{a}=\frac{\left(p+\lambda_{b}\right) \lambda_{1} C_{0}}{p^{3}+a p^{2}+b p+c} \\
w_{c}=\frac{\left(p+\lambda_{a}\right)\left(p+\lambda_{b}\right) C_{0}}{p^{3}+a p^{2}+b p+c} \\
w_{b}=\frac{\left(p+\lambda_{a}\right) \lambda_{4} C_{0}}{p^{3}+a p^{2}+b p+c} \\
w_{g}=\frac{\lambda_{5}}{p+\lambda_{g}} \cdot \frac{\left(p+\lambda_{a}\right) \lambda_{4} C_{0}}{p^{3}+a p^{2}+b p+c}
\end{array}
$$

where

$$
\begin{gathered}
a=\lambda_{a}+\lambda_{b}+\lambda_{c} \\
b=\lambda_{a} \lambda_{b}+\lambda_{b} \lambda_{c}+\lambda_{c} \lambda_{a}-\lambda_{1} \lambda_{2}-\lambda_{3} \lambda_{4} \\
c=\lambda_{a} \lambda_{b} \lambda_{c}-\lambda_{1} \lambda_{2} \lambda_{b}-\lambda_{3} \lambda_{4} \lambda_{a}
\end{gathered}
$$


To obtain the Lallace inversion for (A2a) through (A2d) using available formulae we first rewrite the denominator into:

$$
p^{3}+a p^{2}+b p+c=\left(p+k_{1}\right)\left(p+k_{2}\right)\left(p+k_{3}\right)
$$

Calculations using parameters in a large range have shown that we can calculate the three constants as follows:

$$
\begin{gathered}
k_{1}=a / 3-2 \sqrt{-\beta} \cos (\theta / 3) \\
k_{2}=\frac{a-k_{1}+\sqrt{\left(a-k_{1}\right)^{2}-4 c / k_{1}}}{2} \\
k_{3}=\frac{a-k_{1}-\sqrt{\left(a-k_{1}\right)^{2}-4 c / k_{1}}}{2} \\
\theta=\cos ^{-1}\left(\frac{\alpha}{-\beta \sqrt{-\beta}}\right) \\
\alpha=-\frac{a^{3}}{27}+\frac{a b}{6}-\frac{c}{2} \\
\beta=\frac{b}{3}-\frac{a^{2}}{9}
\end{gathered}
$$

The dimensions for $k_{1}, k_{2}$ and $k_{3}$ are the same: [ $\left.\mathrm{T}^{-1}\right]$. The above solutions are based on the following assumptions:

$$
\alpha^{2}+\beta^{3}<0 ; \quad \& \quad\left(a-k_{1}\right)^{2}-4 c / k_{1}>0
$$

Calculations have shown that these assumptions are valid for most practical cases. If we substitute (A4) into (A2a) through (A2d) we can obtain:

$$
\begin{aligned}
& w_{a}=\lambda_{1} C_{0} \cdot \frac{p+\lambda_{b}}{\left(p+k_{1}\right)\left(p+k_{2}\right)\left(p+k_{3}\right)} \\
& w_{c}=C_{0} \cdot \frac{p^{2}+\left(\lambda_{a}+\lambda_{b}\right) p+\lambda_{a} \lambda_{b}}{\left(p+k_{1}\right)\left(p+k_{2}\right)\left(p+k_{3}\right)}
\end{aligned}
$$




$$
\begin{gathered}
w_{b}=\lambda_{4} C_{0} \cdot \frac{p+\lambda_{a}}{\left(p+k_{1}\right)\left(p+k_{2}\right)\left(p+k_{3}\right)} \\
w_{g}=\lambda_{4} \lambda_{5} C_{0} \cdot \frac{p+\lambda_{a}}{\left(p+k_{1}\right)\left(p+k_{2}\right)\left(p+k_{3}\right)\left(p+\lambda_{g}\right)}
\end{gathered}
$$

Applying the general Laplace inversion formula in Erdelyi $^{10}$ (1954) to (A7a) through (A7d), we can obtain the final solutions given in (17a) through (17d). 